\title{
Comparative evaluation of sump drainage by trocar puncture, percutaneous catheter drainage versus operative drainage in the treatment of Intra-abdominal abscesses: a retrospective controlled study
}

\author{
Guosheng Gu${ }^{1 \dagger}$, Jianan Ren, MD, FACS ${ }^{1 *}$, Song Liu' ${ }^{1,2+}$, Guanwei Li', Yujie Yuan ${ }^{3}$, Jun Chen ${ }^{1}$, Gang Han ${ }^{1,4}$, \\ Huajian Ren ${ }^{1}$, Zhiwu Hong ${ }^{1}$, Dongsheng Yan ${ }^{1}$, Xiuwen $\mathrm{Wu}^{1}$, Ning $\mathrm{Li}^{1}$ and Jieshou Li ${ }^{1}$
}

\begin{abstract}
Background: Intra-abdominal and pelvic abscesses are common and result from various illnesses. Percutaneous drainage applies limitedly to well-localized abscesses with appropriate density while surgical drainage usually causes significant physiological disturbance. We herein illustrated an innovative choice "sump drainage with trocar puncture" for the management of intra-abdominal abscesses and compare it with conventional percutaneous and surgical drainage in terms of clinical outcomes and prognosis.

Methods: Medical records of a total of 75 patients with abscesses were retrospectively retrieved and scrutinized. Data consisted of demographics, abscesses characteristics and treatment outcomes including postoperative complication, duration of hospitalization, postoperative recurrence of abscesses, subsequent surgery, ultimate stoma creation and survival rate. All enrolled patients were divided into trocar group $(n=30)$, percutaneous group $(n=20)$ and surgical group $(n=25)$ according to the therapeutic modalities. One-way ANOVA and $t$-test with Welch's correction were used in continuous variables, and Chi-squared test as well as Fisher's exact test for categorical variables. The cumulative incidence of subsequent surgery and ultimate stoma creation was also indicated by the Kaplan-Meier method and compared by log-rank test.
\end{abstract}

Results: The risk of ultimate stoma creation $(p=0.0069)$ and duration of postoperative hospitalization $(p=0.0077)$ were significantly decreased in trocar group compared with the surgical group. Patients receiving trocar puncture also tended to be less likely to have subsequent surgery $(p=0.097)$. Patients in trocar group displayed a lower rate of postoperative complication than the percutaneous $(p=0.0317)$ and surgical groups $(p=0.0175)$. As for Kaplan-Meier analysis, the cumulative incidence of ultimate stoma creation of the patients using sump drainage was also significantly different among three groups during follow-up period $(p=0.011)$.

Conclusion: This novel technique "sump drainage by trocar puncture" could produce better clinical outcomes and prognosis than conventional percutaneous drainage and surgical intervention. It might become an optimal choice in the management of intra-abdominal abscesses in the future.

Keywords: Percutaneous catheter drainage, Operative drainage, Sump drainage, Trocar puncture, Intra-abdominal abscesses

\footnotetext{
* Correspondence: JiananR@gmail.com

${ }^{\dagger}$ Equal contributors

'Department of Surgery, Jinling Hospital, Medical School of Nanjing

University, 305 East Zhongshan Road, Nanjing 210002, China

Full list of author information is available at the end of the article

of the article
}

() Biomed Central

(c) 2015 Gu et al.; licensee BioMed Central. This is an Open Access article distributed under the terms of the Creative Commons Attribution License (http://creativecommons.org/licenses/by/2.0), which permits unrestricted use, distribution, and reproduction in any medium, provided the original work is properly credited. The Creative Commons Public Domain Dedication waiver (http://creativecommons.org/publicdomain/zero/1.0/) applies to the data made available in this article, unless otherwise stated. 


\section{Background}

Intra-abdominal and pelvic abscesses are common and result from various illnesses, such as trauma, surgery and Crohn's disease [1]. It remains a challenge to clinical doctors as it may result in severe and complicated intraabdominal and systemic infection together with a high recurrence rate.

Over the last several decades, percutaneous catheter drainage $(\mathrm{PCD})$ has been recommended as a primary choice for source control, and surgical intervention is required for percutaneously inaccessible abscesses or abscesses complicated with perforated diseased bowel [2]. However, the relatively narrow catheter that can only provide simple suction without irrigation function in conventional PCD technique dramatically hampers its application in clinical practice. In most cases, patients with viscous pus, cellulitis, necrotic tissues and especially polycystic abscesses have to receive laparotomy. Surgery is apparently the most direct therapeutic measure to control intra-abdominal and pelvic abscesses, the procedure of which depends on the anatomical source of infection, the degree of peritoneal inflammation, the generalized septic response and the patients' general conditions [3]. However, the surgical treatment is far more invasive that leads to the more physiologic upset for the patients, and appears to have a higher mortality rate in contrast with the percutaneous approach [4].

To solve this problem, we advocated an innovative device named "sump drain" which is placed under the guidance of trocar puncture to replace the traditional small-diameter catheter in PCD method. Our previous studies $[5,6]$ have reported that this technique providing continuous irrigation and suction function was scarcely blocked by tissues, pus or coagulates and therefore appropriate for the treatment of intra-abdominal abscesses, especially those abscesses that are contraindications for PCD technique.

This study was designed to compare clinical outcomes and prognosis between sump drainage by trocar puncture, conventional PCD and surgical drainage (SD) in the management of intra-abdominal abscesses.

\section{Methods}

\section{Participants and definitions}

We retrospectively collected all patients registered in our unit between January 2006 and February 2012. Patients who were diagnosed having an intra-abdominal or pelvic abscess would be included in this study. The definition of intra-abdominal and pelvic abscesses was extraluminal enhancing fluid collections $1 \mathrm{~cm}$ or greater in diameter confirmed by CT scan or ultrasound, with purulence documented at the time of drainage [7]. The exclusion criteria consisted of no treatment of intra-abdominal or pelvic abscess, perianal abscesses and less than 3 months of follow-up period.

In current study, we classified the cause of all abscesses as four main types. 1) Trauma: abscess appeared immediately after trauma or was discovered by CT or ultrasound before operation. 2) Surgery: abscesses emerged within 1 month after surgery that could be due to tumor, obstruction or even trauma. 3) Crohn's disease and 4) Intestinal tuberculosis: patients who were diagnosed as Crohn's disease/intestinal tuberculosis and never underwent trauma and surgery would be considered into this category.

All enrolled patients were from the same medical care team in our institution. All operations, including trocar puncture, PCD and SD, were performed by the same surgeon. Prior to the procedures, the risks and benefits of each treatment were discussed with every patient, and informed consent was obtained.

\section{Data collection}

For each participant, we collected the following variables: age, sex, APACHE II score before treatment of abscesses, date of treatment, abscess size and location measured by CT scan or ultrasound, date of discharge and bacteria culture result of purulence samples. The abscess size was determined by the maximum diameter of the abscess.

Our primary outcome was the rate of subsequent surgery and ultimate stoma creation. Secondary endpoints were postoperative complication, duration of hospitalization, recurrence of abscess, and survival condition. The subsequent surgery only included abdominal surgery after abscess treatment and excluded stoma reversal operation. The postoperative recurrence of abscess was defined as the development of an enhancing fluid collection at the same site as the initial lesion occurring within 1 month after the treatment of abscess. Postoperative recurrence of abscess was considered as a separate parameter, instead of being included in postoperative complications.

\section{Management}

For all patients, broad-spectrum antibiotics were prescribed on diagnosis of abscesses and adjusted subsequently according to the bacteria culture result of purulence samples. As a first line therapy in our institution, enteral nutrition was given initially for the majority of enrolled patients with the normal physiological function of the bowel. For patients who failed to tolerate enteral nutrition or suffered intestinal dysfunction, parenteral nutrition was prescribed temporarily. Enteral nutrition would be tried again when the patients' condition improved, and be established eventually [8].

In the current study, three strategies were used in the management of abscesses. The PCD was defined as procedure using an aspiration and insertion of a conventional 
drainage catheter. Briefly speaking, after local anesthesia the collection was punctuated with an 18-gauge sheath needle using ultrasound or CT guidance. Then a guidewire was inserted and an 8-F to $16-\mathrm{F}$ pigtail catheter was advanced and sutured to the skin. The size of the catheter depends on the maximum transverse diameter of the abscesses.

The SD was defined as operation with abscess drainage or for exploration, with or without concomitant bowel resection $[9,10]$. Surgical options included debridement of necrotic tissue, resection or suture of a diseased or perforated viscus, resection of ischemic bowel and repair/ resection of traumatic perforations. Laparotomy was usually performed through a midline incision and abscesses cavities were routinely evacuated prior to closure of the fascia. The objectives were both to establish the cause of peritonitis and to control the origin of infection.

The trocar puncture with sump drain was an innovative technique that has been described in our previous studies $[5,6]$. A specific device named "sump drain" was used to replace the conventional catheter. This sump drain contains a two-channel drainage. The inner tube of the two-channel has vacuum aspiration, while the outer tube is devised with several holes, thereby providing continuous irrigation and suction function. The general procedures are outlined below.

After evaluating the location and depth of the abscess by CT scan or ultrasound, local anesthesia, skin incision (usually 10-15 $\mathrm{mm}$ ) and blunt dissection of subcutaneous tissues were performed consecutively. Afterwards, a 22gauge needle was punctured for diagnostic localization and aspiration. A 12-mm laparoscopic trocar was then placed into the abscess cavity through the same direction as the needle. A 10-mm sump drain, which was actually a modified closed double-lumen irrigation-suction tube, was inserted through the trocar cannula and then the trocar was removed, leaving the sump drain in situ.

\section{Statistics}

SPSS software (SPSS for Windows, version 19.0, SPSS, Chicago, IL) was used for all statistical analysis. All analysis was two-tailed and p-values $<0.05$ was considered statistically significant. For continuous variables, mean \pm $\mathrm{SD}$ was calculated. One-way ANOVA was performed to compare variance among three groups and students' $t$-test with Welch's correction was used to compare two groups. For categorical variables, chi-squared test was performed to compare the constituent ratio among the three groups, and Fisher's exact test was performed between two groups. Given the time-dependent property of the probabilities of subsequent surgery and ultimate stoma creation, the cumulative incidence was also calculated (one minus the estimate of the survival function) by the Kaplan-Meier method and compared by log-rank test.

\section{Ethics}

This study was approved by the Ethics Committee of Jinling Hospital.

\section{Results}

A total of 75 patients were enrolled and divided into three groups: 30 patients in trocar group, 20 in PCD group and 25 in SD group. The average age was $42.1 \pm$ $15.9,36.0 \pm 15.5$ and $41.2 \pm 17.7$ years, respectively $(\mathrm{p}=$ $0.504)$. A male predominance was observed in all groups ( 21 of 30,11 of 20 and 20 of $25, p=0.194$ ), which was in accordance with our previous epidemiological study ${ }^{10}$. APACHE II score demonstrated a similar disease severity among patients in all groups $(9.50 \pm 6.23$ for trocar group, $9.06 \pm 3.72$ for PCD group and $10.0 \pm 5.25$ for SD group, $\mathrm{p}=0.842)$. In trocar and $\mathrm{PCD}$ groups, surgery was the primary cause of abscesses (15 of 30 and 8 of 20 , respectively) while Crohn's disease was the main reason in SD group (11 of 25). The distribution of causes was showed in Table 1.

The abscess size was similar among all three groups $(7.25 \pm 3.63 \mathrm{~cm}$ in trocar group, $7.56 \pm 3.55 \mathrm{~cm}$ in PCD group and $7.00 \pm 4.04 \mathrm{~cm}$ in $\mathrm{SD}$ group, $\mathrm{p}=0.754)$. Abdominal and pelvic cavities were the most common locations for abscess development in all groups. Psoas and intestinal loop were also predilection sites for abscesses. Multiple abscesses were found in 4, 2 and 2 patients in three groups, respectively (Table 2).

13 samples in trocar group, 9 in PCD group and 14 in SD group were demonstrated to contain Escherichia coli in purulence. Klebsiella pneumoniae followed by Staphylococci aureus were also cultured commonly in trocar, PCD and SD groups. Multiple bacteria were found in 2, 1 and 1 patients in three groups, respectively (Table 2).

Table 1 Demographics and clinical parameters of patients with intra-abdominal abscesses

\begin{tabular}{lllll}
\hline & $\begin{array}{l}\text { Trocar group } \\
(\mathrm{n}=30)\end{array}$ & $\begin{array}{l}\text { PCD group } \\
(\mathrm{n}=20)\end{array}$ & $\begin{array}{l}\text { SD group } \\
(\mathrm{n}=25)\end{array}$ & P-value \\
\hline \multicolumn{1}{c}{ Age (yr.) } & $42.1 \pm 15.9$ & $36.0 \pm 15.5$ & $41.2 \pm 17.7$ & 0.504 \\
Male & 21 & 11 & 20 & 0.194 \\
APACHE II score & $9.50 \pm 6.23$ & $9.06 \pm 3.72$ & $10.0 \pm 5.25$ & 0.842 \\
Cause & & & & 0.372 \\
Trauma & 9 & 5 & 5 & - \\
Surgery & 15 & 8 & 8 & - \\
Crohn's disease & 5 & 5 & 11 & - \\
Intestinal tuberculosis & 1 & 0 & 1 & - \\
Others & 0 & $2^{\mathbf{a}}$ & 0 & \\
\hline
\end{tabular}

apancreatic cancer and B-cell lymphoma 
Table 2 Characteristics of abscesses

\begin{tabular}{lllll}
\hline & $\begin{array}{l}\text { Trocar group } \\
(\mathrm{n}=30)\end{array}$ & $\begin{array}{l}\text { PCD group } \\
(\mathrm{n}=20)\end{array}$ & $\begin{array}{l}\text { SD group } \\
(\mathrm{n}=25)\end{array}$ & P-value \\
\hline Abscess size (cm) & $7.25 \pm 3.63$ & $7.56 \pm 3.55$ & $7.00 \pm 4.04$ & 0.754 \\
Abscess location & & 1 & 2 & 0.980 \\
Psoas & 1 & 1 & 2 & - \\
Interloop & 1 & 14 & 16 & - \\
Abdominal & 19 & 2 & 3 & - \\
Pelvic & 5 & 2 & 2 & - \\
Multiple abscesses & 4 & & & 0.985 \\
Bacteria of purulence & & 9 & 14 & - \\
Escherichia coli & 13 & 4 & 5 & - \\
Klebsiella pneumoniae & 8 & 3 & 2 & - \\
Staphylococci aureus & 4 & 2 & 1 & - \\
Proteus mirabilis & 1 & 1 & 1 & - \\
Multiple & 2 & $1^{\mathbf{b}}$ & $2^{\mathbf{c}}$ & - \\
\hline Others & $2^{\mathbf{a}}$ & & & \\
\hline
\end{tabular}

${ }^{\mathbf{a}}$ Candida albicans and Bacteroides fragilis. ${ }^{\mathbf{b}}$ Enterococcus faecalis

${ }^{C}$ Enterococcus faecalis and Serratia marcescens

The average follow-up period for patients in Trocar, PCD and SD groups was $20.7 \pm 12.5$ months, $14.0 \pm 5.16$ months and $19.1 \pm 8.00$ months, respectively $(\mathrm{p}=0.0525)$. Only 1 patient $(3.33 \%)$ had postoperative complication (pulmonary infection) in trocar group whereas 5 patients $(25.0 \%$; 1 bleeding, 2 wound infection and 2 pulmonary infection) in PCD and 7 patients (28.0\%; 2 bleeding, 3 wound infection,
1 pulmonary infection and 1 intra-abdominal infection) in SD group had postoperative complications. Statistical analysis suggested a significantly decreased incidence of postoperative complication in trocar group than $\mathrm{PCD}$ $(\mathrm{p}=0.0317)$ and SD groups $(\mathrm{p}=0.0175)$. However, statistical difference was not observed between PCD and SD groups $(\mathrm{p}=0.999)$.

Clinical outcomes were indicated in Table 3. In trocar group, 2 patients $(6.67 \%)$ had ultimate stoma creation. However, the rate increased up to $25.0 \%$ (5 patients) and $40.0 \%$ (10 patients) in PCD and SD groups. The risk of ultimate stoma creation was significantly decreased in trocar group compared with the surgical group ( $\mathrm{p}=0.0069) .13$ patients $(43.3 \%)$ in trocar group whereas $12(60.0 \%)$ and 18 (72.0\%) in PCD and SD groups underwent subsequent surgery. Lesion resection and anastomosis was the most common type of subsequent surgery for patients with trocar puncture, and stoma creation was the most common approach for patients with PCD and surgical interventions. Patients in Trocar group tended to be less likely to have subsequent surgery $(\mathrm{p}=0.097)$. As for Kaplan-Meier analysis (Fig. 1), the cumulative incidence of ultimate stoma creation of the patients receiving trocar puncture was also significantly different among three groups during follow-up period $(\mathrm{p}=0.011)$.

The duration of postoperative hospitalization was $15.3 \pm$ 15.2, $23.1 \pm 24.8$ and $55.4 \pm 75.0$ days in trocar, PCD and SD groups respectively. A remarkable decrease of

Table 3 Clinical outcomes of management of intra-abdominal abscesses

\begin{tabular}{|c|c|c|c|c|}
\hline & Trocar group $(n=30)$ & PCD group $(n=20)$ & SD group $(n=25)$ & p-value \\
\hline Follow-up period (month) & $20.7 \pm 12.5$ & $14.0 \pm 5.16$ & $19.1 \pm 8.00$ & 0.0525 \\
\hline Postoperative complication & $1(3.33 \%)$ & $5(25.0 \%)$ & $7(28.0 \%)$ & $0.0316^{a}$ \\
\hline Bleeding & 0 & 1 & 2 & \\
\hline Wound infection & 0 & 2 & 3 & \\
\hline Pulmonary infection & 1 & 2 & 1 & \\
\hline Intra-abdominal infection & 0 & 0 & 1 & \\
\hline Duration of postoperative hospitalization & $15.3 \pm 15.2$ & $23.1 \pm 24.8$ & $55.4 \pm 75.0$ & $<0.0001^{b}$ \\
\hline Postoperative recurrence of abscess & $17(56.7 \%)$ & 15 (75.0 \%) & $15(60.0 \%)$ & 0.399 \\
\hline Subsequent surgery & $13(43.3 \%)$ & $12(60.0 \%)$ & $18(72.0 \%)$ & 0.097 \\
\hline Lesion resection and anastomosis & 5 & 1 & 6 & - \\
\hline Stoma creation & 2 & 5 & 9 & - \\
\hline Right hemicolectomy & 2 & 4 & 2 & - \\
\hline Peritoneal lavage & 3 & 2 & 0 & - \\
\hline Left hemicolectomy & 1 & 0 & 0 & - \\
\hline Subtotal colectomy & 0 & 0 & 1 & \\
\hline Ultimate stoma creation & $2(6.67 \%)$ & $5(25.0 \%)$ & $10(40.0 \%)$ & $0.0127^{c}$ \\
\hline Death & $2(6.67 \%)$ & $3(15.0 \%)$ & $2(8.00 \%)$ & 0.588 \\
\hline
\end{tabular}

${ }^{\mathrm{a}}$ Trocar vs. PCD, $\mathbf{p}=\mathbf{0 . 0 3 1 7}$; Trocar vs. SD, $\mathbf{p}=\mathbf{0 . 0 1 7 5}$; $\mathrm{PCD}$ vs. SD, $\mathrm{p}=0.999$

${ }^{\mathbf{b}}$ Trocar vs. $\mathrm{PCD}, \mathrm{p}=0.199 ;$ Trocar vs. SD, $\mathbf{p}=\mathbf{0 . 0 0 7 7} ; \mathrm{PCD}$ vs. $\mathrm{SD}, \mathbf{p}=\mathbf{0 . 0 3 3}$

cTrocar vs. PCD, $\mathrm{p}=0.100$; Trocar vs. SD, $\mathbf{p}=\mathbf{0 . 0 0 6 9}$; $\mathrm{PCD}$ vs. SD, $\mathrm{p}=0.352$ 


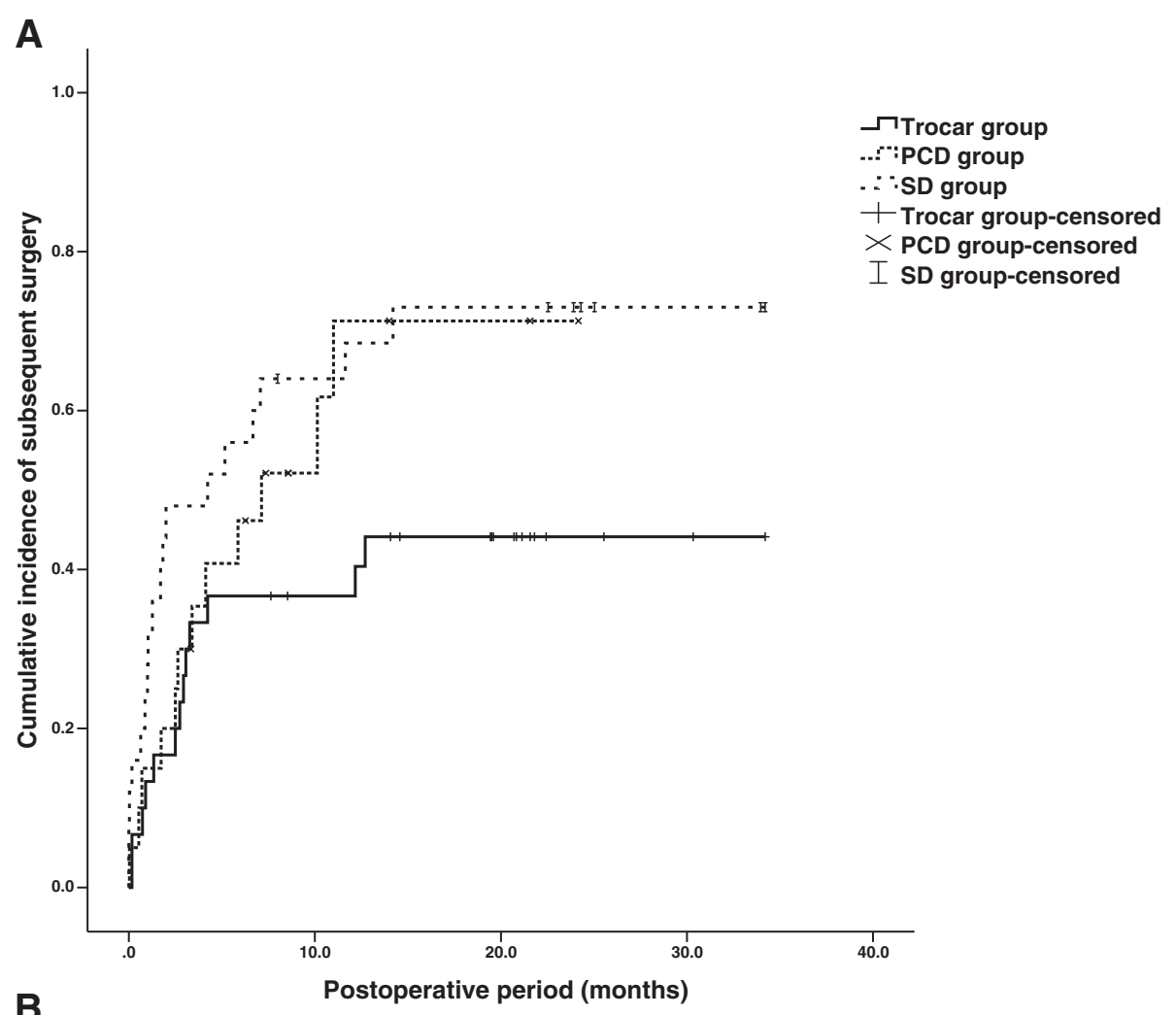

B

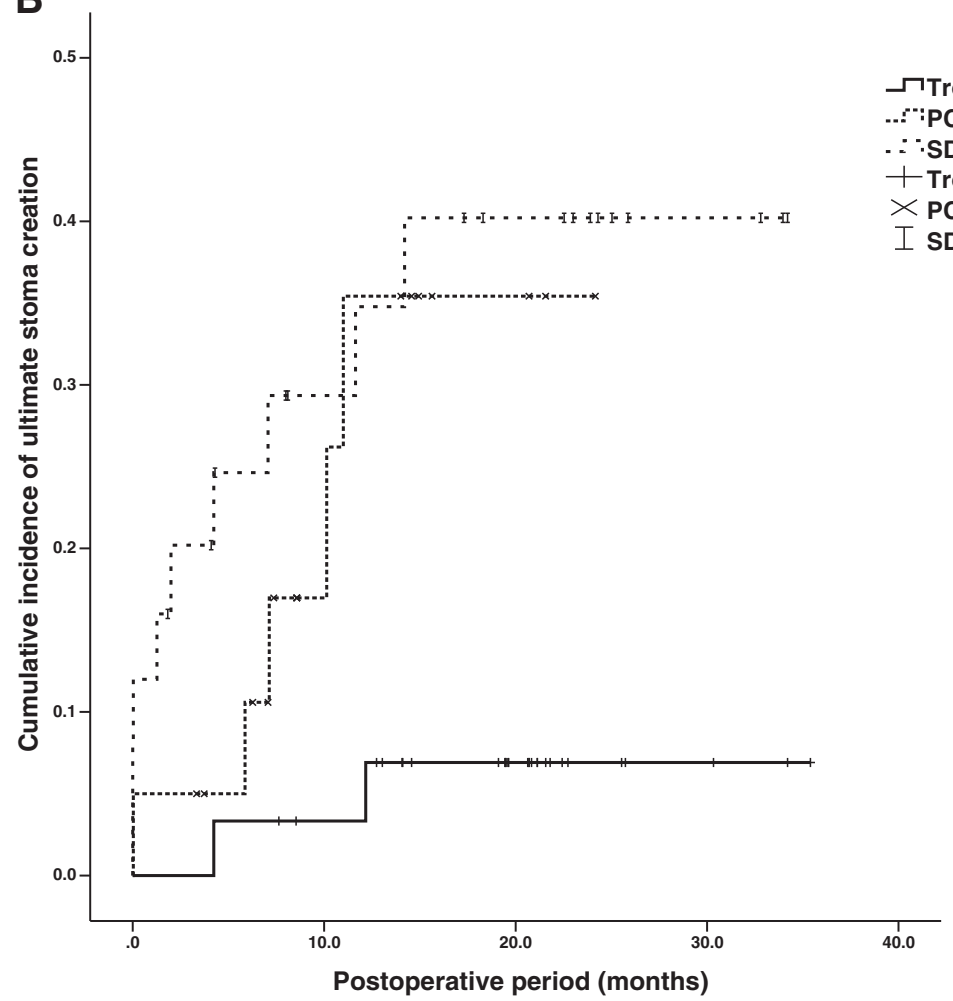

Fig. 1 Cumulative incidence of subsequent surgery among the patients in Trocar, PCD and SD groups (A). Patients in Trocar group tended to have a lower incidence of subsequent surgery during follow-up period $(p=0.070)$. Cumulative incidence of ultimate stoma creation among the patients in Trocar, PCD and SD groups (B). The cumulative incidence of ultimate stoma creation of the patients receiving trocar puncture was significantly different among three groups during follow-up period $(p=0.011)$. Cumulative incidence is one minus the estimate of the survival function 
postoperative hospital stay existed in trocar group compared with SD group $(\mathrm{p}=0.0077)$. Meanwhile, the duration of postoperative hospitalization in $\mathrm{PCD}$ group was also shorter than that in SD group $(\mathrm{p}=0.033)$. Patients using trocar puncture did not have statistically different postoperative duration compared with patients using $\mathrm{PCD}$ technique $(\mathrm{p}=0.199)$.

17 patients $(56.7 \%)$ had postoperative recurrence of abscesses in trocar group while $15(75.0 \%)$ in PCD group and $15(60.0 \%)$ in SD group. The difference of postoperative recurrence had no statistical significance $(\mathrm{p}=0.399)$.

During the follow-up period, 2 patients $(6.67 \%)$ died in trocar group while $3(15.0 \%)$ and $2(8.0 \%)$ died in PCD and SD groups, respectively. Survival rate was similar among all three groups $(\mathrm{p}=0.588)$.

\section{Discussion}

In the current study, we evaluated the clinical outcomes and prognosis of three different strategies in the management of intra-abdominal abscesses. Based on the analysis of 75 eligible patients, our preliminary results suggested several advantages when performing trocar puncture with sump drain instead of traditional PCD and SD. These advantages included decreased rates of ultimate stoma creation and postoperative complication as well as shorter duration of postoperative hospitalization. Moreover, patients with sump drain also tended to have a lower rate of subsequent surgery.

In recent decades, PCD has emerged as a primary option in the management of intra-abdominal abscesses. In the guideline published by Surgical Infection Society and the Infectious Disease Society of America [11], PCD is recommended and preferable to SD in well-localized fluid collection of appropriate density, results in significantly less physiological alteration and reduces the need for open procedure [2]. Open surgical technique is typically considered for poorly localized, complex, or diffuse fluid collections, necrotic tissue, high-density fluid, or percutaneously inaccessible collections. Also, suspicious perforation of diseased bowel with signs of extensive or massive free air requires a laparotomy [2].

Therefore, we can conclude to some extent that there are several hardly-avoidable disadvantages for both PCD and SD. PCD can only be performed on well-localized and low-density abscesses $[12,13]$ while SD may result in a remarkable physiologic alteration, prolonged hospitalization and decelerated recovery process $[14,15]$.

As an innovative drainage technique, trocar puncture with sump drain is being widely used in our clinical practice and has been illustrated in our previous studies $[5,6]$. It has also been confirmed as a promising management option in Crohn's disease patients with lower rate of postoperative complication, shorter duration of postoperative hospitalization and lower rate of ultimate stoma creation (our unpublished data), similar to the results of the present study.

This modified technique uses a larger-diameter tube (sump drain) instead of conventional small size catheter to achieve less blockage and to more rapidly obliterate abscess cavity under the assistance of continuous irrigation and suction function with negative pressure. Moreover, this "two-channel tube" device contains an interval at the ends between inner and outer tubes and several holes in the outer tube, which may further reduce the possibility of blockage by tissues, pus or coagulates. Trocar is employed to assist the sump drain reaching the abscess cavity through percutaneous puncture in order to maintain physiologic stability. As a result, this modified technique can be successfully applied to the drainage of viscous pus, cellulitis, necrotic tissues and especially polycystic abscess that are contraindications of PCD [5].

Based on the findings, we conclude that this modified technique not only expands its indications in clinical application in contrast with PCD, but also achieves better clinical outcomes and prognosis compared with SD.

We admit several limitations in the current study. First of all, as a retrospective controlled study, a potential selection bias may exist. However, patients were assigned into three groups after discussing with the surgeons on the risks and benefits of each treatment and signing consent forms. No specific patients were selected to a certain strategy of drainage in the setting of treatment in our center. As the demographic (such as age and gender distribution), clinical (such as APACHE II score and primary cause) and abscess-related parameters (such as abscess size, location and bacteria of purulence) at baseline were all similar among all groups, we assumed these enrolled patients had been relatively well-matched in this controlled study. Second, only 75 qualified patients were enrolled in this study. The small sample size may hinder the statistical power and conceal the results. Besides, a longer follow-up period is also required in larger and multicenter studies.

\section{Conclusions}

In conclusion, we demonstrated that patients with sump drain had lower rates of ultimate stoma creation and postoperative complication as well as shorter duration of postoperative hospitalization compared with those using $\mathrm{PCD}$ or SD. This innovative technique may emerge as an optimal choice in the management of intra-abdominal abscesses.

Abbreviations

PCD: Percutaneous catheter drainage; SD: Surgical drainage.

\section{Competing interests}

The authors declare that they have no competing interests. 


\section{Authors' contributions}

Study conception and design: GS, JA; Acquisition of data: GS, SL, YJ, XW, JC, GH; Analysis and interpretation of data: GS, SL, HJ, ZW, DS; Drafting of manuscript: GS, SL, GW; Critical revision: JA, NL, JS. All authors read and approved the final manuscript.

\section{Acknowledgements}

This study was supported by grants from Research Talents of Jiangsu Province, China (BRA2011232) and National Natural Science Foundation of China (81270478).

\section{Author details}

'Department of Surgery, Jinling Hospital, Medical School of Nanjing University, 305 East Zhongshan Road, Nanjing 210002, China. ${ }^{2}$ Center for the Study of Inflammatory Bowel Disease, Harvard Medical School, Massachusetts General Hospital, Boston, MA, USA. ${ }^{3}$ Department of Gastrointestinal-Pancreatic Surgery, the First Affiliated Hospital of Sun Yat-sen University, Guangzhou, China. ${ }^{4}$ Department of General Surgery, Second Affiliated Hospital of Jilin University General Surgery, Center of Jilin University, Changchun, China.

\section{Received: 2 July 2013 Accepted: 4 May 2015}

\section{Published online: 09 May 2015}

\section{References}

1. Mazuski JE, Solomkin JS. Intra-abdominal infections. Surg Clin N Am. 2009:89:421-+.

2. Solomkin JS, Mazuski J. Intra-abdominal sepsis: newer interventional and antimicrobial therapies. Infect Dis Clin North Am. 2009;23:593-+.

3. Sartelli M. A focus on intra-abdominal infections. World JEmerg Sur. 2010;5.

4. Sirinek KR. Diagnosis and treatment of intra-abdominal abscesses. Surg Infect. 2000:1:31-8.

5. Liu S, Ren J, Gu G, Yuan Y, Han G, Wu Y, et al. Drainage of intra-abdominal abscesses with gastrointestinal fistula in Crohn's disease using a sump drain via trocar puncture: a modified strategy. Tech Coloproctol. 2013;17:459-61.

6. Gu G, Ren J, Yuan Y, Chen J, Fan Y, Cao S, et al. An innovative technique for intra-abdominal abscess drainage using a sump drain by trocar puncture. Am Surg. 2011;77:E166-7.

7. Nguyen DL, Sandborn WJ, Loftus Jr EV, Larson DW, Fletcher JG, Becker B, et al. Similar outcomes of surgical and medical treatment of intra-abdominal abscesses in patients with Crohn's disease. Clin Gastroenterol Hepatol. 2012:10:400-4.

8. Liu S, Ren J, Hong Z, Yan D, Gu G, Han G, et al. Efficacy of erythropoietin combined with enteral nutrition for the treatment of anemia in Crohn's disease: a prospective cohort study. Nutr Clin Pract. 2013;28:120-7.

9. Feagins LA, Holubar SD, Kane SV, Spechler SJ. Current strategies in the management of intra-abdominal abscesses in Crohn's disease. Clin Gastroenterol Hepatol. 2011;9:842-50.

10. Xie Y, Zhu W, Li N, Li J. The outcome of initial percutaneous drainage versus surgical drainage for intra-abdominal abscesses in Crohn's disease. Int J Colorectal Dis. 2012;27:199-206.

11. Solomkin JS, Mazuski JE, Bradley JS, Rodvold KA, Goldstein EJC, Baron EJ, et al. Diagnosis and management of complicated intra-abdominal infection in adults and children: guidelines by the surgical infection society and the infectious diseases society of America. Clin Infect Dis. 2010;50:133-64.

12. Theisen J, Bartels H, Weiss W, Berger H, Stein HJ, Siewert JR. Current concepts of percutaneous abscess drainage in postoperative retention. J Gastrointest Surg. 2005:9:280-3.

13. Bufalari A, Giustozzi G, Moggi L. Postoperative intraabdominal abscesses: Percutaneous versus surgical treatment. Acta Chir elg. 1996;96:197-200

14. van Ruler $\mathrm{O}$, Mahler CW, Boer KR, Reuland EA, Gooszen HG, Opmeer BC, et al. Comparison of on-demand vs planned relaparotomy strategy in patients with severe peritonitis - A randomized trial. JAMA. 2007;298:865-73.

15. Maher MM, Gervais DA, Kalra MK, Lucey B, Sahani DV, Arellano R, et al. The inaccessible or undrainable abscess: How to drain it. Radiographics. 2004;24:717-35.

\section{Submit your next manuscript to BioMed Central and take full advantage of:}

- Convenient online submission

- Thorough peer review

- No space constraints or color figure charges

- Immediate publication on acceptance

- Inclusion in PubMed, CAS, Scopus and Google Scholar

- Research which is freely available for redistribution 